\title{
BMJ
}

\section{Community involvement in dengue vector control: cluster randomised trial}

\author{
V Vanlerberghe, research fellow, ${ }^{1}$ M E Toledo, epidemiologist, ${ }^{2}$ M Rodríguez, entomologist, ${ }^{3}$ D Gomez, \\ epidemiologist, ${ }^{3}$ A Baly, economist, ${ }^{2}$ I R Benitez, director, ${ }^{3}$ P Van der Stuyft, head of department
}

${ }^{1}$ Epidemiology and Disease Control Unit, Public Health Department, Institute of Tropical Medicine, Nationalestraat 155, 2000 Antwerp, Belgium

${ }^{2}$ Department of Epidemiology, Institute of Tropical Medicine

"Pedro Kouri", Ciudad de La Habana, Cuba

${ }^{3}$ Provincial Center of Surveillance and Vector Control, Guantanamo, Cuba

Correspondenceto:VVanlerberghe vvanlerberghe@itg.be

Cite this as: BMJ 2009;338:b1959 doi:10.1136/bmj.b1959

\section{ABSTRACT}

Objective To assess the effectiveness of an integrated community based environmental management strategy to control Aedes aegypti, the vector of dengue, compared with a routine strategy.

Design Cluster randomised trial.

Setting Guantanamo, Cuba.

Participants 32 circumscriptions (around 2000 inhabitants each).

Interventions The circumscriptions were randomly allocated to control clusters $(n=16)$ comprising routine Aedes control programme (entomological surveillance, source reduction, selective adulticiding, and health education) and to intervention clusters ( $n=16)$ comprising the routine Aedes control programme combined with a community based environmental management approach. Main outcome measures The primary outcome was levels of Aedes infestation: house index (number of houses positive for at least one container with immature stages of Ae aegypti per 100 inspected houses), Breteau index (number of containers positive for immature stages of $\mathrm{Ae}$ aegypti per 100 inspected houses), and the pupae per inhabitant statistic (number of Ae aegypti pupae per inhabitant).

Results All clusters were subjected to the intended intervention; all completed the study protocol up to February 2006 and all were included in the analysis. At baseline the Aedes infestation levels were comparable between intervention and control clusters: house index $0.25 \% v 0.20 \%$, pupae per inhabitant $0.44 \times 10^{-3} v$ $0.29 \times 10^{-3}$. At the end of the intervention these indices were significantly lower in the intervention clusters: rate ratio for house indices 0.49 (95\% confidence interval 0.27 to 0.88 ) and rate ratio for pupae per inhabitant 0.27 (0.09 to 0.76).

Conclusion A community based environmental management embedded in a routine control programme was effective at reducing levels of Aedes infestation.

Trial registration Current Controlled Trials ISRCTN88405796.

\section{INTRODUCTION}

Forty per cent of the world's population are at risk of dengue, ${ }^{1}$ an important mosquito borne viral disease. Each year dengue causes 24000 deaths, 250000-
500000 cases of haemorrhagic fever, and up to $50 \mathrm{mil}$ lion cases of dengue fever. ${ }^{23}$ The global burden of dengue for the year 2001 was estimated to be 528000 disability adjusted life years (DALYs). Dengue is responsible for an annual average loss of 658 DALYs per million population in Latin America and the Caribbean and is of the same order of magnitude as tuberculosis in this region. ${ }^{45}$ Its importance to public health is growing rapidly as a result of a 30 -fold increase in incidence $^{6}$ following the geographical expansion of its main vector, Aedes aegypti, since the $1960 \mathrm{~s}^{3}$ and to the accrued cocirculation of multiple serotypes, which increase the risk of sequential infection with the dengue virus and severity of disease. ${ }^{2}$

No specific antiviral treatment or vaccine against dengue is available. The prevention of lethality hinges on early detection and supportive treatment of severe cases. Prevention of transmission is crucial to decrease the burden of dengue, and control of Aedes is the only available strategy. For the past few decades spraying of outdoor spaces has been the main method of control, directed against adult mosquitoes. This method is of questionable efficacy and is often inefficiently applied in the community. ${ }^{57}$ More recently, insecticide impregnated curtains and covers for domestic water containers showed promising results on vector densities. ${ }^{8}$ Vector control methods directed against the immature Aedes stages, such as environmental management, larvicides, copepods, Bacillus thuringiensis toxins, or insect growth regulators are increasingly used in routine programmes, with variable success rates; this variability often results from the absence of active involvement of the community. ${ }^{5}$

The plea for community participation in environmental management strategies is plausible on theoretical grounds, as the presence, or at least the density, of Ae aegypti depends on human behaviour. Notwithstanding, evidence on the effectiveness of community based Aedes control is weak and controversial owing to, among others, methodological shortcomings in the published studies, such as short follow-up periods, questionable study designs, and evaluation of outcomes by proxy indicators. ${ }^{910}$ Community involvement strategies vary with respect to target groups and intervention procedures ${ }^{11-14}$ but are implemented at 
the level of geographical or administrative areas; for the purpose of an effectiveness evaluation they should be set up as cluster randomised controlled trials. ${ }^{15}$ To date this has not been done. ${ }^{9}$ Also, the Ae aegypti larval indices, classically used to measure entomological effects - the house, container, and breteau index - do not necessarily reflect adequately the risk of dengue transmission and it has been argued that pupae per inhabitant is a more appropriate measure of the abundance of adult vectors. ${ }^{116}$

We assessed the effectiveness of integrated community based environmental management (domiciliary and communal) compared with routine Aedes control in reducing pupal statistics as well as traditional $A e$ aegypti larval indices.

\section{METHODS}

We carried out a cluster randomised controlled trial in Guantanamo, a city with 243000 inhabitants in western Cuba and with an average temperature of $31^{\circ} \mathrm{C}$ and an average rainfall of $610 \mathrm{~mm} /$ year concentrated in a short wet season (April-July). Guantanamo, together with Santiago de Cuba and Havana, have the highest $A e$ aegypti infestation levels in the country (house indices up to $1.73 \%$ in 1997-2004). These can be attributed mainly to a deficient water supply, the bad condition or absence of covers on water storage containers, and a lack of adequate environmental management. Guantanamo was affected by the dengue epidemics of $1981^{17}$ and $2001-2 .^{18}$

\section{Study design}

In September 2004, 32 "circumscriptions" (the most decentralised geopolitical unit, comprising about 500 houses and 2000 inhabitants) were selected in central urban Guantanamo. In January 2005, after obtaining approval from the community, the circumscriptions were randomly allocated to 16 control clusters and to 16 intervention clusters by drawing numbers from a bag. In the control clusters the routine Aedes control programme was implemented throughout the study period; in the intervention clusters it was combined with the tested strategy. Sample size was calculated as proposed for cluster randomized trials. ${ }^{19} \mathrm{We}$ aimed to detect a $50 \%$ reduction in the house indices, with a power of $80 \%$ and an $\alpha$ error of 0.05 , assuming a coefficient of variation (standard deviation divided by the mean) of 0.25 for the clusters' house index. The trial

Key elements of intervention

- Discussion on the intervention with relevant local stakeholders and formation of a local steering committee

- Creation of formal task forces (community working groups) at grassroots level to secure community involvement in environmental management

- Establishment of coordination mechanisms between community working groups, health services, and local government structures to strengthen intersectoral coordination

- Harmonisation of the intervention and the action plan of the local vector control programme was designed to last until the end of 2007, with an interim analysis in February 2006. No firm stopping rules were defined.

\section{Control and intervention clusters}

In the 16 control clusters the routine Aedes control programme was implemented throughout the study period. This programme is vertically organised but leaves some room for decentralised decision making. The programme's vector control workers have no fixed area of responsibility and cover the municipality on a rotational basis. They carried out standard control activities: entomological surveillance and source reduction through periodic inspection of houses (in cycles of 11 days), larviciding of water storage containiners with temephos, selective adulticiding with cypermethrin or clorpyriphos when Ae aegypti foci were detected, communication and education on dengue prevention, and enforcement of mosquito control legislation by imposing fines.

In the 16 intervention clusters, external researchers from the Institute of Tropical Medicine "Pedro Kouri," Cuba, and the Institute of Tropical Medicine, Belgium, assisted the local health authorities in Guantanamo to set up a community based environmental management strategy that complemented the routine vector control programme.

The key elements of intervention (box) were derived from best practices in two pilot studies on community participation in dengue control in Havana and Santiago de Cuba. ${ }^{20-23}$

The discussion process with relevant stakeholders was supported by formative research (focus group discussions with grassroots actors and in-depth interviews with formal leaders and health staff) in October to December 2004. This resulted in fine tuning the intervention to its local context. A local steering committee with epidemiologists, entomologists, social scientists, and educational professionals was set up and headed by the provincial director of the vector control programme. The committee was responsible for implementing the intervention, coaching community working groups, organising training sessions according to the needs of the grassroots actors involved, coordinating with the local health authorities, and documenting the process of implementation. The external research group was responsible for development of the study protocol and quality control and provided technical support during bimonthly visits.

In January 2005 the formal grassroots task forces, called Grupo de Trabajo Comunitario (community working group), were created in each of the 16 intervention circumscriptions. They became the driving force for the intervention by actively involving the community and securing intersectoral support links. A community working group was composed of 10 to 20 members: formal and informal leaders, public health workers from the vector control programme, and a nurse from the neighbourhoods' family medicine practice. Members of the community working group did not receive financial incentives, but 
Table 1| Household characteristics in intervention and control clusters, October 2004, Guantanamo, Cuba. Values are numbers (percentages) unless stated otherwise

\begin{tabular}{|c|c|c|}
\hline Characteristic & Intervention clusters & Control clusters \\
\hline No of randomly sampled households & 400 & 400 \\
\hline Mean (SD) No of inhabitants per household & $3.93(1.95)$ & $3.93(2.01)$ \\
\hline \multicolumn{3}{|l|}{ Type of housing: } \\
\hline House & $367(92)$ & $346(87)$ \\
\hline Apartment & $22(6)$ & $35(9)$ \\
\hline Room & $11(3)$ & $19(5)$ \\
\hline \multicolumn{3}{|l|}{ Water provision point: } \\
\hline Inside house & $287(72)$ & $270(68)$ \\
\hline Outside house & $103(26)$ & $122(31)$ \\
\hline Communal well or water truck & $10(2)$ & $8(2)$ \\
\hline \multicolumn{3}{|l|}{ Frequency of water distribution: } \\
\hline Continuous or every day & $102(26)$ & $119(30)$ \\
\hline Alternate days & $144(36)$ & $95(24)$ \\
\hline Every $3-5$ days & $124(31)$ & $145(36)$ \\
\hline Every $\geq 6$ days & $28(7)$ & $33(8)$ \\
\hline Irregular (water truck) & $2(1)$ & $8(2)$ \\
\hline \multicolumn{3}{|l|}{ Mean (SD) No and types of water storage containers per household: } \\
\hline Ground level container & $1.80(1.43)$ & $1.83(1.37)$ \\
\hline Cistern & $0.49(1.14)$ & $0.31(0.77)$ \\
\hline Buckets and other small deposits & $0.47(1.75)$ & $0.42(1.53)$ \\
\hline \multicolumn{3}{|l|}{ Main methods used to control mosquito nuisance: } \\
\hline Electric fan & $338(85)$ & $341(85)$ \\
\hline Bed net & $134(34)$ & $141(35)$ \\
\hline Smoke and fumes & $36(9)$ & $46(12)$ \\
\hline Knowledge that dengue is vector borne disease & $360(90)$ & $344(86)$ \\
\hline Correct knowledge of at least one measure to prevent dengue & $396(99)$ & $398(99)$ \\
\hline \multicolumn{3}{|c|}{ Presence of risk factors for Aedes proliferation in and around the home: } \\
\hline Water storage containers not covered during day & $146(37)$ & $145(36)$ \\
\hline Badly covered water storage containers & $120(30)$ & $113(28)$ \\
\hline Water storage containers in bad condition & $102(25)$ & $94(24)$ \\
\hline Incorrect use of larvicides* & $225(56)$ & $242(61)$ \\
\hline
\end{tabular}

participatory training sessions were organised with them on needs assessment, social mobilisation, and the elaboration and evaluation of action plans.

From February 2005 onwards each community working group carried out a situation assessment with the community, identified local needs and priorities for environmental and dengue control, and elaborated action plans. These action plans varied between circumscriptions but contained activities such as locally designed social communication intending to mobilise the population and change behaviour (for example, to cover water storage containers correctly, to protect artificial containers, not to remove larvicide from water storage containers); negotiations with the community and with governmental intersectoral groups to eliminate environmental risks outside the domiciliary environment (constructing evacuation systems for waste water, repairing broken water pipelines, improving communal waste collection); contracting a local manufacturer to produce covers for water storage containers from used beer cans or wood and nylon, which were sold to the households at a low price $(\mathrm{Cu} \$ 5$; equivalent to $£ 0.13, € 0.20$ or $\$ 0.24$ at the time of study); surveillance of environmental risks with locally produced and periodically updated maps; and visits by teams of community members to houses with repeated Aedes infestation. Implementation of the action plans in the intervention clusters started in April 2005. Implementation relied on community and routine programme resources. Only the reproduction of locally designed leaflets and posters was partially financed by research funds.

Simultaneously, a well defined and fixed area of responsibility was assigned to individual vector control workers to strengthen their relationship with the community and to assure an optimal inclusion of the community based strategy in the vector control programme.

\section{Data collection}

In November 2004 a baseline survey was carried out on a systematic random sample of 800 households to assess knowledge, attitudes, and practices regarding dengue and its prevention, socioeconomic characteristics, and environmental risks in and around dwellings. 
In January 2006, 12 group discussions with 118 inhabitants and 16 group discussions with the community working groups were held in the intervention clusters to assess perceptions on actual and preintervention involvement of the community.

Members of the national vector control programme carried out routine entomological surveys in cycles of 11 days in all dwellings of the municipality. This provided the entomological information for all clusters by cycle and by house block for the period January 2005 to February 2006: number of houses inspected, number of wet containers (any container with water-for example, containers used to store water or non-utility containers such as waste bins that become filled with rain water) by type, number of houses and containers positive for immature stages of Ae aegypti, distribution of immature stages, and absolute number of pupae. The data combine the observations of the routine vector control workers and of the quality control inspectors, who revisited a systematic sample of 33\% of the houses.

\section{Data analysis}

We carried out a descriptive analysis of the baseline survey. The members of the local research team analysed the transcripts of group discussions and relevant documents describing the intervention process. The analysis was guided by the five criteria proposed by Rifkin for appraising community participation: needs identification, leadership, organisation, resource mobilisation, and management. ${ }^{24}$ For every cluster a consensus score from 1 to 5 (1=none, $2=$ weak, $3=$ fair, $4=$ good, and $5=$ excellent) was assigned to each criterion. The distribution of the scores per criteria for all intervention clusters was summarised by the median and range. To obtain a measure of participation in each intervention cluster we averaged its scores.

The primary outcome was levels of Aedes infestation. We calculated, per cluster and per cycle, house index (number of houses positive for at least one container with immature stages of $A e$ aegypti per 100 inspected houses), Breteau index (number of containers positive for immature stages of Ae aegypti per 100 inspected

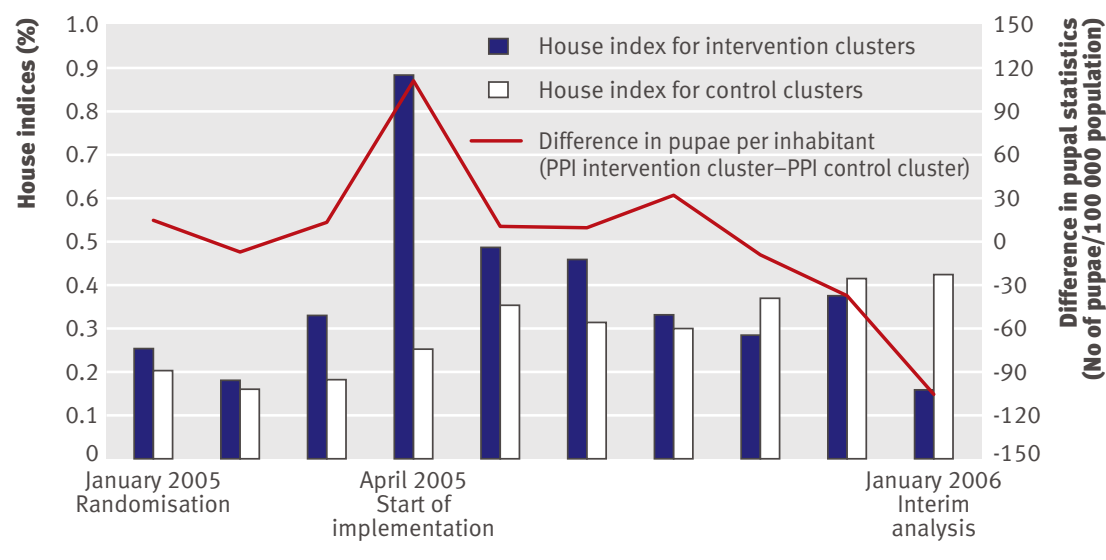

House indices in intervention and control clusters and difference in pupae per inhabitant between clusters, January 2005 to February 2006, Guantanamo, Cuba houses), and pupae per inhabitant (number of Ae aegypti pupae per inhabitant).

A crude mid-term analysis in February 2006 showed a positive effect of the intervention. In view of this, and soaring entomological indices in Guantanamo municipality as a whole, the provincial health authorities decided to stop the trial and to generalise the intervention strategy to the whole city. Hence the preintervention period was defined as the three cycles covering January 2005 and the end of intervention period as the three cycles covering January 2006. To evaluate the effect of intervention on the house and Breteau indices and pupae per inhabitant we constructed generalised linear random effect regression models with negative binomial link function. We evaluated the time effect (preintervention and end of intervention) and group effect (intervention or control) at cycle by cluster level. This model takes into account the nature of the data (repeated measures in each cluster) and allows the assessment of a possible interaction between time effect and group effect, capturing the effect of the intervention on the outcomes.

A descriptive graph was elaborated to illustrate the evolution of the entomological indices. We calculated the mean house indices and pupae per inhabitant for three inspection cycles in each cluster and then averaged these values for the control and intervention groups.

We computed the proportion of breeding sites that were positive for first and second instar larvae for each cycle and each cluster and averaged these by intervention and control group for the preintervention period and end of intervention period. We assessed the percentage of blocks with repeated positivity during the preintervention period and end of intervention period. The influence of intervention on these secondary outcome measures was evaluated by a $\chi^{2}$ test. We used Stata 9 and SPSS 15.0 for analyses.

\section{RESULTS}

All clusters received the intended intervention; they completed the study protocol up to February 2006 and were included in the analysis. Overall, there were 8422 houses and 33688 inhabitants in the intervention clusters and 10748 houses and 42992 inhabitants in the control clusters. Baseline characteristics were similar between the clusters except for a higher frequency of water distribution in the intervention clusters (table 1). In all houses at least one environmental or behavioural risk factor was observed. Overall, $78 \%$ of the intervention households and $76 \%$ of the control households perceived that the activities realised by the vector control workers were necessary, and 13\% and $11 \%$, respectively, remembered that a positive breeding site had been found in the past.

In January 2006 community involvement in the intervention clusters was assessed as "fair" (average overall score 3.34) compared with almost non-existent before intervention. The median score for the needs identification and leadership criteria was 4 and for the other criteria was 3 . For all criteria the variability 
between clusters was high. The highest score per cluster was 4.8 (close to excellent involvement) and the lowest was 1.4 (close to no involvement). Ten clusters were identified as good strategy adaptors (score $\geq 3$ ) and six as poor strategy adaptors (score $<3$ ).

\section{Entomological outcome measures}

At baseline the entomological indices were comparable between the intervention and control clusters (figure and table 2). At the start of the intervention, when the inspection of potential breeding sites by the routine vector control worker was intensified with the support of the community, the house index peaked and thereafter gradually declined. In the control clusters a steady increasing trend over time was observed. In January 2006, infestation levels in the intervention clusters were significantly lower than those in the control clusters (table 2) $-50 \%$ lower for the Breteau and house indices and 73\% lower for pupae per inhabitant. The predominant breeding sites for both clusters remained the water storage containers at ground level (70-75\%).

The proportion of early immature stages (first and second instar larvae) increased significantly more in the intervention clusters (9\% preintervention, $43 \%$ end of intervention) than in the control clusters $(6 \%$ and $12 \% ; \mathrm{P}=0.004)$. In the intervention area non-significant $(\mathrm{P}=0.3)$ decrease in the percentage of repeatedly positive blocks $(5.8 \% v 3.5 \%)$ compared with a significant increase $(\mathrm{P}=0.005)$ in the control area $(13.2 \%$ and $17.0 \%)$ was observed.

\section{DISCUSSION}

After one year Aedes foci were reduced to levels almost $50 \%$ lower in clusters where the community based environmental management strategy was embedded in the routine programme, compared with clusters that had the routine control programme alone. The difference in the number of pupae per inhabitant, a recommended indicator to measure the abundance of adult vector and the risk of dengue transmission, ${ }^{25}$ reached 73\%. Early immature stages (first and second instar larvae) were more common at the end of intervention, which indicates that breeding sites were eliminated more promptly with involvement of the community.

One of the main strengths of our study, compared with earlier work, ${ }^{11-1326-28}$ was the use of a cluster randomised controlled design taking into account possible confounding by ecological, climatic, and other unknown factors influencing Aedes infestation. ${ }^{15}$

Entomological indices and statistics were the outcome measures. We added the pupae per inhabitant statistic to the traditional Ae aegypti larval indices used in most Aedes control studies as it reflects better the abundance of adult vectors and has a more direct relation with risk of dengue transmission. ${ }^{25}$ Surveillance of clinical cases of dengue has been found inadequate to monitor transmission, ${ }^{29}$ but IgM seroconversion (in young children) would, theoretically, be a better outcome measure than entomological indices. However, cluster randomised trials of interventions to control Aedes need huge sample sizes to attain sufficient power to show an effect on seroconversion, given the relative low incidence of dengue infection, its cross reactivity with other flaviviridae infections, and the short duration of IgM seropositivity ${ }^{30}$; and additionally pose serious operational challenges. Furthermore, it is hardly feasible to measure an effect on transmission in Cuba, since dengue occurs only in sporadic outbreaks. In fact, the routine surveillance system did not pick up any dengue activity in the Guantanamo province during the study period.

The close involvement of the provincial vector control programme constitutes a possible methodological limitation from a theoretical point of view. This could, admittedly, have resulted in some improved quality of routine work; punctual initiatives by individual control area workers mimicking intervention activities cannot be completely excluded either. The routine vector control activities, which obviously could not be interrupted, were closely monitored and were found comparable in both the control and the intervention areas. However, such "contamination," if any, would produce only an underestimate of the true intervention effect.

Likewise we had to rely on entomological data collected through the routine surveillance system (with concomitant removal of immature stages) organised in 11 day cycles. Apart from possible non-differential underestimation of the number of breeding sites, the real limitation here is that methods and procedures were no longer fully standardised after the start of the intervention. The motivation of the routine workers in the intervention clusters increased (as such, a desirable secondary effect), and so did the motivation in the corresponding communities. Inhabitants became more

Table 2 |Entomological indices in control and intervention clusters, 2005-6, Guantanamo, Cuba. Values are means (standard deviations) unless stated otherwise

\begin{tabular}{|c|c|c|c|c|c|c|c|}
\hline \multirow[b]{2}{*}{ Indices } & \multicolumn{3}{|c|}{ Preintervention } & \multicolumn{4}{|c|}{ End of intervention } \\
\hline & $\begin{array}{l}\text { Intervention } \\
\text { clusters }\end{array}$ & Control clusters & $\begin{array}{l}\text { Rate ratio intervention: } \\
\text { control* }^{*}(95 \% \mathrm{Cl})\end{array}$ & $\begin{array}{l}\text { Intervention } \\
\text { clusters }\end{array}$ & Control clusters & 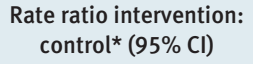 & P value* \\
\hline House index (\%) & $0.25(0.20)$ & $0.20(0.17)$ & $1.45(0.78$ to 2.70$)$ & $0.26(0.21)$ & $0.48(0.45)$ & 0.49 (0.27 to 0.88$)$ & 0.018 \\
\hline $\begin{array}{l}\text { Breteau index (per } 100 \\
\text { houses) }\end{array}$ & $0.27(0.23)$ & $0.20(0.17)$ & 1.55 (0.83 to 2.87 ) & $0.28(0.25)$ & $0.52(0.52)$ & 0.48 (0.26 to 0.88$)$ & 0.016 \\
\hline $\begin{array}{l}\text { Pupae per inhabitant } \\
\left(\times 10^{-3}\right)\end{array}$ & $0.44(0.54)$ & $0.29(0.42)$ & 1.67 (0.76 to 3.69$)$ & $0.36(0.51)$ & $1.40(1.90)$ & 0.27 (0.09 to 0.76$)$ & 0.013 \\
\hline
\end{tabular}

*Estimated with a generalised linear random effect regression model with negative binomial link function. 


\section{WHAT IS ALREADY KNOWN ON THIS TOPIC}

Dengue prevention is mainly based on the control of its vector, Aedes aegypti

As previous vector control strategies showed variable success rates, effective and sustainable alternatives are awaited by policy makers

Community participation has been advocated for dengue control, but evidence from cluster randomised controlled trials is lacking

\section{WHAT THIS STUDY ADDS}

Community based environmental management integrated in a routine dengue prevention and control programme can reduce levels of Aedes infestation by $50-75 \%$ compared with a routine programme as a single strategy

willing to cooperate with the vector control workers in their routine search for immature mosquitoes in and around dwellings and together they found "hidden" breeding sites. This observation bias explains the peak level in all entomological indices in the intervention clusters at the start of intervention. We have no hard data to substantiate that such differential observation did not fade over time, but key informants indicate that it was, in essence, maintained. If this were the case, the reductions in entomological indices observed between April 2005 and January 2006 in the intervention clusters would reflect real decreases-just as real as the increases in the control clusters. Also, the difference between control and intervention clusters in the number of pupae per inhabitant estimated in January 2006 would be an underestimate of the intervention effect. If, on the contrary, the search for breeding sites had returned to being comparable in all clusters, the observed difference in January 2006 would reflect the true intervention effect.

In February 2006, before we could sort this matter out, the provincial health authorities decided on the basis of a crude interim analysis to extend the intervention strategy to the whole city of Guantanamo. This led de facto to the end of the formal trial. At that moment the community involvement in the environmental management was not yet homogeneous over the intervention clusters, as involving the community takes time and is not a spontaneous activity. A suitable formal organisation must be identified or set up to guide the community involvement strategy, ${ }^{1331}$ and members of these organisations need training. ${ }^{32}$ Then, the opportunity must be given for initiative and autonomous action. Furthermore, institutionalisation of the approach is crucial for continuity of actions and for the sustainability of the strategy. ${ }^{32}$ In Guantanamo we also secured integration of the bottom-up approach into the top-down programme, as advocated by previous research, ${ }^{33}$ by involving the provincial director in the design of the strategy, by assigning the routine vector control workers to specified fixed areas, by taking into account feedback of community working groups to adjust the activities of the vertical programme, and by establishing links between the community working group and the government sectors represented at local level. Such integration was possible only because the existing vertical vector control programme was already functioning well. Another influencing factor, described previously ${ }^{31}$ is a favourable political and sociocultural context that supports discussion of issues affecting the wellbeing of individuals and the community, acquisition of knowledge, and active community involvement in implementation of the programme.

Some previous small or non-randomised or uncontrolled studies of equal (or shorter) duration had already suggested positive effects of community based dengue control programmes (exclusively or in combination with other vector control methods)." These cover a broad range of activities. In our study the action plans of all intervention clusters included the targeting of ground level water storage containers and exterior artificial deposits. The strategy described by Kay and $\mathrm{Nam}^{26}$ in Vietnam, a large scale (but uncontrolled) study, is comparable to the approach adopted in Guantanamo except for the use of copepods instead of temephos, and resulted in the absence of dengue cases in 32 communes during 2002-5.

The approach used in Guantanamo was principally inspired by a strategy implemented in Santiago, which, in a quasi-experimental set-up, had equal effectiveness as an intensified routine programme. ${ }^{23}$ By adapting this intervention to the specific context of Guantanamo and formally testing it, we showed not only the effectiveness of its main strategic components in other areas with relatively low infestation levels, but also, and possibly more importantly, its transferability. Whether similar or even better results can be obtained in areas with higher Aedes infestation remains to be studied. Finally, stopping the trial early was unfortunate from a scientific perspective but from a public health perspective we achieved perhaps the most relevant result possible: health authorities appraised the innovative strategy to be successful and feasible and decided to extend it first to the whole city and subsequently to the whole province. Besides, the ongoing scaling-up provides a unique opportunity to study the influence of acknowledged determinants of successful project extension such as the nature of innovation, attributes of the health system, implementation strategies, and the larger social system reaction ${ }^{34}$ in the context of participation in Aedes control.

We thank the health sector staff involved in the dengue prevention and control activities, the people of Guantanamo who participated in the study, and A Huys for editing support.

Contributors: VV, MET, and PVdS conceived and designed the study. MET JRB, and $A B$ supervised field activities and data collection. RM and GD carried out the fieldwork. VV and PVdS did the statistical analysis. All authors contributed to the interpretation of the results and revised subsequent drafts of the manuscript. PVdS and MET are guarantors. All authors declare, as researchers, independence from the funders. Funding: This study received partial funding from the framework agreement between the Institute of Tropical Medicine and the Belgian Directorate-General for Development Co-operation (project No 95900) The funding source was not involved in any stage of the research process Competing interests: None declared.

Ethical approval: This study was approved by the ethical committee of the Institute of Tropical Medicine "Pedro Kouri" and from the national health authorities. Community representatives approved the intervention, and 
individual informed consent was obtained from interviewees and from inhabitants of the inspected houses.

1 Farrar J, Focks D, Gubler D, Barrera R, Guzman MG, Simmons C, et al. Towards a global dengue research agenda. Trop Med Int Health 2007;12:695-9.

2 Deen JL, Harris E, Wills B, Balmaseda A, Hammond SN, Rocha C, et al. The WHO dengue classification and case definitions: time for a reassessment. Lancet 2006;368:170-3.

3 Gibbons RV, Vaughn DW. Dengue: an escalating problem. BMJ 2002;324:1563-6.

4 Shepard DS, Suaya JA, Halstead SB, Nathan MB, Gubler DJ, Mahoney RT, et al. Cost-effectiveness of a pediatric dengue vaccine. Vaccine 2004;22:1275-80.

5 TDR for research on diseases of poverty. Report of the Scientific Working Group meeting on Dengue, 1-5 Oct, 2006. Geneva: WHO. (TDR/SWG/08 ed.)

6 World Health Organization. Dengue and dengue hemorrhagic fever. In: Kindhauser MK, ed. Communicable diseases 2002: global defense against the infectious disease threat. Geneva, WHO, 2003:140-3. (WHO/CDS/2003.15.)

7 Newton EA, Reiter P. A model of the transmission of dengue fever with an evaluation of the impact of ultra-low volume (ULV) insecticide applications on dengue epidemics. Am J Trop Med Hyg 1992;47:709-20.

8 Kroeger A, Lenhart A, Ochoa M, Villegas E, Levy M, Alexander N, et al. Effective control of dengue vectors with curtains and water containe covers treated with insecticide in Mexico and Venezuela: cluster randomised trials. BMJ 2006;332:1247-52.

9 Heintze C, Garrido MV, Kroeger A. What do community-based dengue control programmes achieve? A systematic review of published evaluations. Trans R Soc Trop Med Hyg 2007;101:317-25.

10 Perez D, Lefevre P, Sanchez L, Van der Stuyft P. Comment on: What do community-based dengue control programmes achieve? A systematic review of published evaluations. Trans $R$ Soc Trop Med Hyg 2007;101:630-1.

11 Espinoza-Gomez F, Hernandez-Suarez CM, Coll-Cardenas R. Educational campaign versus malathion spraying for the control of Aedes aegypti in Colima, Mexico. J Epidemiol Community Health 2002;56:148-52.

12 Lloyd LS, Winch P, Ortega-Canto J, Kendall C. Results of a communitybased Aedes aegypti control program in Merida, Yucatan, Mexico. Am J Trop Med Hyg 1992;46:635-42.

13 Nam VS, Nguyen TY, Tran VP, Truong UN, Le OM, Le VL, et al. Elimination of dengue by community programs using Mesocyclops (Copepoda) against Aedes aegypti in central Vietnam. Am J Trop Med Hyg 2005;72:67-73.

14 Parks W, Lloyd L, Nathan MB, Hosein E, Odugleh A, Clark GG, et al. International experiences in social mobilization and communication for dengue prevention and control. Dengue Bull 2005;28(special suppl):1-7.

15 Ukoumunne OC, Gulliford MC, Chinn S, Sterne JA, Burney PG, Donner A. Methods in health service research. Evaluation of health interventions at area and organisation level. BMJ 1999;319:376-9.

16 Nathan MB, Focks DA, Kroeger A. Pupal/demographic surveys to inform dengue-vector control. Ann Trop Med Parasitol 2006;100(suppl 1):S1-3.
17 Kouri GP, Guzman MG, Bravo JR, Triana C. Dengue haemorrhagic fever/dengue shock syndrome: lessons from the Cuban epidemic, 1981. Bull World Health Organ 1989;67:375-80.

18 Guzman MG, Pelaez O, Kouri G, Quintana I, Vazquez S, Penton M, et al. [Final characterization of and lessons learned from the dengue 3 epidemic in Cuba, 2001-2002]. Rev Panam Salud Publica 2006;19:282-9.

19 Hayes RJ, Bennett S. Simple sample size calculation for clusterrandomized trials. Int J Epidemiol 1999;28:319-26.

20 Baly A, Toledo ME, Boelaert M, Reyes A, Vanlerberghe V, Ceballos E, et al. Cost effectiveness of Aedes aegypti control programmes: participatory versus vertical. Trans $R$ Soc Trop Med Hyg 2007;101:578-86.

21 Perez D, Lefevre P, Sanchez L, Sanchez LM, Boelaert M, Kouri G, et al. Community participation in Aedes aegypti control: a sociological perspective on five years of research in the health area " 26 de Julio", Havana, Cuba. Trop Med Int Health 2007;12:664-72.

22 Sanchez L, Perez D, Perez T, Sosa T, Cruz G, Kouri G, et al. Intersectoral coordination in Aedes aegypti control. A pilot project in Havana City, Cuba. Trop Med Int Health 2005;10:82-91.

23 Toledo ME, Vanlerberghe V, Baly A, Ceballos E, Valdes L, Searret M, et al. Towards active community participation in dengue vector control: results from action research in Santiago de Cuba, Cuba. Trans R Soc Trop Med Hyg 2007;101:56-63.

24 Rifkin SB, Muller F, Bichmann W. Primary health care: on measuring participation. Soc Sci Med 1988;26:931-40.

25 Focks DA, Chadee DD. Pupal survey: an epidemiologically significant surveillance method for Aedes aegypti: an example using data from Trinidad. Am J Trop Med Hyg 1997;56:159-67.

26 Kay B, Nam VS. New strategy against Aedes aegypti in Vietnam. Lancet 2005;365:613-7.

27 Leontsini E, Gil E, Kendall C, Clark GG. Effect of a community-based Aedes aegypti control programme on mosquito larval production sites in El Progreso, Honduras. Trans R Soc Trop Med Hyg 1993;87:267-71.

28 Winch PJ, Leontsini E, Rigau-Perez JG, Ruiz-Perez M, Clark GG, Gubler DJ. Community-based dengue prevention programs in Puerto Rico: impact on knowledge, behavior, and residential mosquito infestation. Am J Trop Med Hyg 2002;67:363-70.

29 Kay BH, Nam VS, Tien TV, Yen NT, Phong TV, Diep VT, et al. Control of aedes vectors of dengue in three provinces of Vietnam by use of Mesocyclops (Copepoda) and community-based methods validated by entomologic, clinical, and serological surveillance. Am J Trop Med Hyg 2002;66:40-8.

30 Vordam V, Kuno G. Laboratory diagnosis of dengue virus infections In: Guber DJ, Kuno G, eds. Dengue and dengue hemorrhagic fever. London: CAB International, 1997:313-34.

31 Zakus JD, Lysack CL. Revisiting community participation. Health Policy Plan 1998;13:1-12.

32 Toledo Romani ME, Vanlerberghe V, Perez D, Lefevre P, Ceballos E, Bandera D, et al. Achieving sustainability of community-based dengue control in Santiago de Cuba. Soc Sci Med 2007;64:976-88.

33 Gubler DJ, Clark GG. Community involvement in the control of Aedes aegypti. Acta Trop 1996;61:169-79.

34 Simmons R, Brown J, Diaz M. Facilitating large-scale transitions to quality of care: an idea whose time has come. Stud Fam Plann 2002;33:61-75.

Accepted: 21 January 2009 